PROCEEDINGS OF THE

AMERICAN MATHEMATICAL SOCIETY

Volume 51, Number 1, August 1975

\title{
MINIMAL $N$-COMPACT SPACES
}

\section{K. P. S. BHASKARA RAO AND ASIT BARAN RAHA}

ABSTRACT. In this note it is established that minimal $N$-compact spaces are compact.

1. Introduction. Given two spaces $X$ and $E$, we say that $X$ is $E$-completely regular (respectively $E$-compact) provided that $X$ is homeomorphic to a subspace (respectively, closed subspace) of $E^{m}$ for some cardinal number $m$ [4]. When $E=R$, the space of real numbers, $E$-compact spaces are precisely the realcompact spaces of Hewitt. When $E=N$, the space of natural numbers with discrete topology, $E$-compact spaces are called $N$-compact spaces.

For any topological property $\pi$ and a nonvoid set $X$, let $\pi(X)$ denote the set of topologies on $X$ with property $\pi . \pi(X)$ is obviously partially ordered under inclusion. A topological space $(X, \mathfrak{T})$ is minimal $\pi$ if $\mathfrak{T}$ is a minimal element in $\pi(X),(X, \mathfrak{T})$ is said to be $\pi$-closed if $\mathfrak{T}$ has property $\pi$ and $X$ is a closed subspace of every $\pi$ space in which it can be embedded. Our problem is to characterise minimal $E$-compact spaces for the case $E=N$. For $E=R$, the problem was solved in [5].

2. Definitions. A subset of a topological space $X$ which is both open and closed is called a clopen subset of $X . B(X)$ denotes the collection of all clopen subsets of $X$.

A 0-dimensional space is a Hausdorff space (sufficient to assume $T_{0}$ ) which has a base for the topology consisting of clopen sets.

A clopen filter $\mathcal{F}$ on a space $X$ is a nonempty collection of clopen subsets of $X$ such that:

(i) $\varnothing \notin \mathcal{F}$;

(ii) if $U, V \in \mathcal{F}$ then $U \cap V \in \mathcal{F}$; and

(iii) $V \in \mathcal{F}, U \in \mathfrak{B}(X)$ and $U \supset V$, then $U \in \mathcal{F}$. A clopen filter $\mathcal{F}$ is fixed if $\bigcap \mathcal{F} \neq \varnothing$ and $\mathcal{F}$ is said to have the countable

Received by the editors July 26, 1973. $54 \mathrm{~F} 45$.

AMS (MOS) subject classifications (1970). Primary 54D25; Secondary 54D35,

Key words and phrases. E-compact, $E$-completely regular, $N$-compact, clopen ultrafilter with countable intersection property. 
intersection property provided that for each countable subcollection $\mathcal{G}$ of $\mathcal{F}$, $\bigcap \oint \neq \varnothing$. A clopen ultrafilter is a clopen filter which is maximal in the collection of all clopen filters ordered by inclusion.

3. Result. In order to prove our result we shall appeal to the following characterisation of $N$-compact spaces due to Herrlich [3] and Chew [2].

3.1. Theorem. A 0-dimensional space $X$ is N-compact if and only if every clopen ultrafilter on $X$ with the countable intersection property is fixed.

3.2. Theorem. The following are equivalent for an $N$-compact space $X$ :

(i) $X$ is minimal N-compact;

(ii) $X$ is $N$-compact-closed;

(iii) $X$ is compact $T_{2}$.

Proof. (i) $\Rightarrow$ (ii). Suppose $(X, \mathcal{T})$ is minimal $N$-compact but it is not $N$-compact-closed. Then there exists an $N$-compact space $Y$ with $X \neq \bar{X} \subset Y$ i.e., $X$ is not a closed subset of $Y$. Suppose $p \in \bar{X}-X(\bar{X}$ denotes the closure of $X$ in $Y$ ). Then $p$ is an accumulation point of $X$. Let $\mathcal{C}=$ $\{V \cap X: V$ is a clopen nbhd of $p$ in $Y\}$. Note that $\cap \mathcal{C}=\varnothing$. Fix $x_{0} \in X$. Define a topology $\mathcal{S}$ on $X$ by the following open neighbourhood bases:

$\mathcal{S}(x)=$ clopen nbhds of $x$ in $(X, \mathcal{J}), x \neq x_{0}$;

$\mathcal{S}\left(x_{0}\right)=\left\{C \cup G: C \in \mathcal{C}\right.$ and $G$ is any clopen nbhd of $x_{0}$ in $\left.\mathfrak{T}\right\}$.

(1) $(X, \mathcal{S})$ is 0 -dimensional. Let $C \cup G \in \mathcal{S}\left(x_{0}\right)$. Then it is already open in $\mathcal{S}$. Again, $(C \cup G)^{c}=C^{c} \cap G^{c}$, a clopen set in $(X, \mathcal{J})$ and belongs to $\mathcal{S}(x)$ for any $x \in C^{c} \cap G^{c}$ and hence is open. So $C \cup G$ is a clopen set in $\mathcal{S}$. Take $x \neq x_{0}$ and $U \in \mathcal{S}(x)$. We have to exhibit an $\mathcal{S}$-clopen set inside $U$. Without loss of generality let $x_{0} \notin U$. Choose $C \in \mathcal{C}$ such that $x, x_{0} \notin C$. Now $C \cup U^{c} \in \mathcal{S}\left(x_{0}\right)$ and is clopen in $\mathcal{S}$. So, $U \cap C^{c}$ contains $x$ and is contained in $U$. Also it is clopen in $\mathcal{S} .(X, \mathcal{S})$ is thus proved to have a base consisting of clopen sets. We shall have to show that $\mathcal{S}$ is Hausdorff. It suffices to show that $\mathcal{S}$ is $T_{0}$. Let $x$ and $y$ be two distinct points of $X$. If $x$ and $y$ are both different from $x_{0}$, then there is nothing to show. So, let us assume $y \neq x=x_{0}$. Since $(X, \mathcal{T})$ is 0 -dimensional there exists a $\mathcal{T}$-clopen set $G$ with $x_{0} \in G$ and $y \notin G$. Again, since $\cap \mathcal{C}=\varnothing$, it is possible to find $C \in \mathcal{C}$ such that $y \notin C$. Then $C \cup G \in \mathcal{S}\left(x_{0}\right)$ by definition and $y \notin(C \cup G)$. Thus $(X, \mathcal{S})$ is $T_{0}$. The 0 -dimensionality of $(X, \mathcal{S})$ is now proved.

(2) $(X, \mathcal{S})$ is strictly weaker than $(X, \mathcal{J})$. Since $Y$ is a 0 -dimensional space, there exists a clopen nbhd $V_{0}$ of $p$ in $Y$ such that $x_{0} \notin V_{0}$. Then $V_{0}^{c} \cap X\left(=\left(Y-V_{0}\right) \cap X\right)$ is a $\mathcal{T}$-clopen nbhd of $x_{0}$. 
Claim. $V_{0}^{c} \cap X \notin \mathcal{S}$. Since $(X, \delta)$ is 0 -dimensional and since $x_{0} \epsilon$ $V_{0}^{c} \cap X \in \mathcal{S}$, there should exist an $\mathcal{S}$-clopen set of the form $C_{1} \cup G_{1}$ such that $x_{0} \in C_{1} \cup G_{1} \subset V_{0}^{c} \cap X$. Then $C_{1} \subset V_{0}^{c} \cap X \subset V_{0}^{c}$. But $C_{1}=V_{1} \cap X$ where $V_{1}$ is a clopen nbhd of $p$ in $Y$. Hence

$$
\varnothing=C_{1} \cap V_{0}=V_{1} \cap X \cap V_{0}=X \cap\left(V_{1} \cap V_{0}\right)
$$

-a contradiction.

(3) $(X, \delta)$ is $N$-compact. Let $\mathcal{U}$ be an ultrafilter of $\delta$-clopen sets with countable intersection property. Because of Theorem 3.1 we shall show that $\bigcap \mathcal{\varnothing} \neq$. If $x_{0} \in \bigcap \mathcal{U}$, nothing to prove. Let $x_{0} \notin \bigcap \mathcal{U}$. Then there exists $U \in \mathcal{U}$ such that $x_{0} \notin U$. Without loss of generality $U$ can be taken to be of the form $X-(C \cup G)=(C \cup G)^{c}$ where $C \cup G \in \mathcal{S}\left(x_{0}\right)\left[x_{0} \in U^{c}\right.$. So there exists $C \cup G \in \mathcal{S}\left(x_{0}\right)$ such that $x_{0} \in C \cup G \subset U^{c}$. Therefore, $x_{0} \notin(C \cup G)^{c}$ $\supset U$ and $(C \cup G)^{c}$ is clopen in $\mathcal{S}$. So $(C \cup G)^{c} \in \mathcal{U}$ by definition of $\mathcal{U}$.]

Let $\mathcal{F}$ be an ultrafilter of $\mathcal{T}$-clopen sets containing $\mathcal{U}$. If $\mathcal{F}$ has countable intersection property then $\mathcal{F}$ will be fixed because of Theorem 3.1 as $(X, \mathcal{T})$ is $N$-compact. Then $\mathcal{U}$ will be fixed also. Let $\left\{F_{n}: n \geq 1\right\}$ be a countable subfamily of $\mathcal{F}$. Write $F_{n} \cap U=H_{n}$ (say). Then $H_{n}=F_{n} \cap U=$ $F_{n} \cap(C \cup G)^{c}$. So

$$
H_{n}^{c}=F_{n}^{c} \cup C \cup G=C \cup\left(F_{n}^{c} \cup G\right) \in \mathfrak{S}\left(x_{0}\right) .
$$

In fact $C \cup\left(G \cup F_{n}^{c}\right)$ is clopen in $\mathcal{S}$. So $H_{n}$ is $\delta$-clopen for each $n$. Let $D \in \mathcal{U}$. Then $H_{n} \cap D \neq \varnothing$ for all $n$. Thus inasmuch as $\mathcal{U}$ is an ultrafilter of clopen sets, $H_{n} \in \mathcal{U}$ for all $n$. Therefore, since $\mathcal{U}$ has countable intersection property, $\bigcap_{n=1}^{\infty} H_{n} \neq \varnothing$ i.e., $\bigcap_{n=1}^{\infty}\left(F_{n} \cap U\right) \neq \varnothing$ or $\left(\bigcap_{n=1}^{\infty} F_{n}\right) \cap U \neq \varnothing$. In particular $\bigcap_{n=1}^{\infty} F_{n} \not \varnothing$. Thus $\mathcal{F}^{\prime}$ has countable intersection property, so $\mathcal{U}$ is fixed. Hence $(X, \mathcal{S})$ is $N$-compact as it is already proved to be 0 -dimensional.

Now $(X, \mathcal{S})$ is $N$-compact and $\mathcal{S}$ is strictly weaker than $\mathcal{T}$. So the minimality of the $N$-compact space $(X, \mathcal{T})$ is contradicted. We therefore conclude that $X=\bar{X}$ i.e., $X$ is closed in $Y$.

(ii) $\Rightarrow$ (iii). This follows from the fact that every $N$-compact space $X$ has a 0 -dimensional compactification; e.g., $\left.\beta_{\{0,1}\right\}^{X}$.

(iii) $\Rightarrow$ (i). Suppose $(X, \mathfrak{T})$ is a compact $N$-compact space. Then it is well known that $(X, \mathfrak{T})$ is minimal Hausdorff. Since each $N$-compact space is $T_{2},(X, \mathfrak{T})$ must be minimal $N$-compact. Q.E.D.

4. Remarks. The results of $[5]$ and this paper may lead one to conjecture that for any $E$, a minimal $E$-compact space is compact. However, this is not 
the case. Let $E$ be the countable minimal Hausdorff space which is not compact [1]. Then $E$ is clearly minimal E-compact but not compact.

\section{REFERENCES}

1. N. Bourbaki, Espaces minimaux et espaces complètement séparés, C. R. Acad. Sci. Paris 212 (1941), 215-218. MR 3, 136.

2. Chew Kim-peu, A characterization of $N$-compact spaces, Proc. Amer. Math. Soc. 26 (1970), 679-682. MR $42 \# 2436$.

3. H. Herrlich, E-kompakte Räume, Math. Z. 96 (1967), 228-255. MR 34 \#5051.

4. S. Mrówka, Further results on E-compact spaces. I, Acta Math. 120 (1968), 161-185. MR $37 \# 2165$.

5. A. B. Raha, Minimal realcompact spaces, Colloq. Math. 24 (1972), fasc. 2, 219-223. MR $46 \# 4482$.

INDIAN STATISTICAL INSTITUTE, 203 B. T. ROAD, CALCUTTA 700035, INDIA 